\title{
Influence of Type la Supernova Environment and Their Peculiar Velocities on Distance measurements
}

\author{
Maria Victorovna Pruzhinskaya*t \\ Lomonosov Moscow State University, Sternberg Astronomical Institute, Universitetsky pr. 13, \\ Moscow 119234, Russia \\ E-mail: pruzhinskaya@gmail.com
}

\section{Pierre-François Léget}

Kavli Institute for Particle Astrophysics and Cosmology, Department of Physics, Stanford

University, Stanford, CA 94305

LPNHE, CNRS/IN2P3, Sorbonne Université, Paris Diderot, Laboratoire de Physique Nucléaire et de Hautes Énergies, F-75005, Paris, France

E-mail: pierrefrancois.leget@gmail.com

Type Ia Supernovae (SNe Ia) are widely used to measure the expansion of the Universe. To perform such measurements the luminosity and cosmological redshift of the SNe Ia have to be determined. The luminosity can be measured thanks to so-called standardization procedure that allows to correlate a luminosity of a supernova with its light curve or spectrum parameters. Unfortunately, even after the standardization procedures there is a remaining dispersion on the Hubble diagram. This dispersion can be due to environmental effects (age, chemical composition, dust around a SN) which are not yet taken into account. Besides the standardization of SN Ia, the Hubble diagram contains another parameter, i.e. the supernova cosmological redshift. In fact the redshift observed on the Earth is also affected by peculiar velocities. The exact contribution of peculiar velocities in the observed redshift is unknown, however it was shown that the peculiar velocities have an impact on the distance measurements and therefore can potentially affect the cosmological analysis. Here, we discuss the impact of SN environment and peculiar velocities on the distance measurements and cosmology. In the epoch of large transient surveys, development of observational techniques and further processing of the data, a study of any possible sources of systematical uncertainties in the cosmological analysis is of high priority.

Accretion Processes in Cosmic Sources - II - APCS2018

3-8 September 2018

Saint Petersburg, Russian Federation

\footnotetext{
* Speaker.

${ }^{\dagger}$ The main results presented here are based on [1,2] papers.
} 


\section{Introduction}

Type Ia Supernovae (SNe Ia) are excellent distance indicators. Observations of distant $\mathrm{SNe}$ Ia led to the discovery of the accelerating expansion of the Universe $[3,4,5,6]$. The most recent analysis of SNe Ia indicates that for a flat $\Lambda \mathrm{CDM}$ cosmology, our Universe is accelerating, with $\Omega_{\Lambda}=0.693 \pm 0.012$ [7].

Cosmological parameters are estimated from the "luminosity distance-redshift" relation of $\mathrm{SNe}$, using the Hubble diagram. Generally, particular attention is paid to standardization of SNe Ia, i.e. to increase of the accuracy of luminosity distance determinations.

In the early studies it was widely assumed that SNe Ia have universal light curves and a constant absolute magnitude at maximum ("standard candles"). This was explained by the similarity of the physical processes that lead to the outburst phenomenon. Generally, the outburst is a thermonuclear explosion of a C-O white dwarf whose mass has become close to or larger than the Chandrasekhar mass. In fact, after the detailed observations of a large number of supernovae had been accomplished, it became clear that the absolute magnitude at maximum can vary within 1 mag. The reasons of luminosity dispersion could be different. First, the mechanism of mass increase by white dwarf is not universal: it can be due to the matter accretion on the white dwarf from the companion star (so-called "single-degenerate (SD) scenario") or the merger of two white dwarfs ("double-degenerate (DD) scenario" or "merging scenario"). Additionally there exist some alternative scenarios, like sub-Chandrasekhar, that is usually associated with weak explosions. These scenarios have internal freedom that results in significant variations in observed light curves of SNe Ia: like point of deflagration-to-detonation transition (for SD scenario), or difference in total mass (for DD scenario). Another important factor which could violate the "standard candle" hypothesis is dust. Dust around the supernovae, as well as in the host galaxy, surely affects light curve behavior. The distribution and properties of dust in host galaxies of supernovae could be different from that in the Milky Way. In addition, the initial chemical composition of the progenitor stars also complicates the picture. A lower metallicity involves an increase of the Chandrasekhar limit and could affect the explosion process. For example, in elliptical galaxies or in halo of spiral galaxies only old, i.e. metal-poor, stars with an age comparable to that of the Universe are located; on the contrary in the star formation regions of spiral galaxies there are young metal-rich stars. The above-listed factors (age, chemical composition of the region around SN, presence of dust) could be considered as environmental effects.

Fortunately, it was established that supernovae are "standardized candles". SNe Ia whose luminosity falls down slowly after the maximum light are brighter than supernovae with a fast decline rate (stretch correction; $[8,9,10])$. Later it was shown that their absolute brightness depends on the color as well (color correction; [11, 12]). However, the origin of this relation remains a big mystery of SN science and is not yet reproduced by radiation-hydrodynamical modelling.

Besides the standardization of SN Ia, the Hubble diagram contains another parameter, i.e. the supernova cosmological redshift. The uncertainty on the redshift is quite often considered negligible. The redshift used in "luminosity distance-redshift" relation is due to the expansion of the Universe assuming Friedman-Lemaitre-Robertson-Walker metric, i.e. the motion within the reference frame defined by the cosmic microwave background radiation (CMB), another words a cosmological redshift. In fact, the redshift observed on the Earth also includes the contribution 
from the Doppler effect induced by radial peculiar velocities due to the Earth's rotational and orbital motions, Galactic rotation, peculiar motion of the Galaxy within the Local Group, "infall" of the Local Group toward the center of the Local Super-cluster, host galaxy peculiar motion etc. The exact contribution of peculiar velocities in the observed redshift is unknown.

To minimize the influence of poorly constrained peculiar velocities in cosmological analyses, usually a $150-300 \mathrm{~km} \mathrm{~s}^{-1}$ peculiar velocity dispersion is added in quadrature to the redshift uncertainty $[13,14]$. It has nonetheless been observed that velocity dispersion can exceed $1000 \mathrm{~km} \mathrm{~s}^{-1}$ in galaxy clusters [15]. If we transform this dispersion into a magnitude error, we will see that for lowredshift region $(z<0.05)$ this error is several times more than the one corresponding to $150 \mathrm{~km} \mathrm{~s}^{-1}$ and $300 \mathrm{~km} \mathrm{~s}^{-1}$, moreover, it is two times larger than the intrinsic dispersion of SNe Ia around the Hubble diagram (0.11 mag, see fig. 2 in [2]). It means that standard methods for peculiar velocity correction do not work for the galaxies inside the clusters and another, more accurate method has to be developed.

The final goal of our work is to study how the environment and peculiar velocities affect the Hubble diagram and to reduce as much as possible the residual dispersion on the Hubble diagram to improve the precision of cosmological parameters.

\section{Environmental effect}

The current cosmological analysis with SNe Ia is based on SALT2 (Spectral Adaptive Light curve Template for Type Ia supernova; [16]) standardization model. This model contains two parameters, $X 1$ describing the time stretching of the light-curve and the colour offset with respect to the average at the date of maximum luminosity in the B-band, $C 1$. SALT2 model is not able to perfectly standardize the luminosity of SNe Ia. However, other standardization methods have been developing, for example, SUGAR (Supernova Useful Generator and Reconstructor; [17]). The main advancement proposed in SUGAR is the addition of two other parameters to characterize the SNe Ia variability. The first depends on the properties of SNe Ia ejecta velocity, the second depends on their calcium lines. The addition of these parameters makes SUGAR the best available model to describe the spectral energy distribution of SNe Ia and improves distance measurements of the order of $15 \%$ relative to the SALT2 method.

To understand the reason of the remaining luminosity variability in standardization models, we investigate how the model parameters correlate with supernovae environment: the galocentric distance and the galaxy morphology (see [1, 18]).

In [1] the host galaxy morphology for 192 SNe Ia from the nearby and SDSS JLA (Joint Lightcurve Analysis, [14]) sample was derived. The supernovae were divided into three subsamples depending on host morphology: elliptical/lenticular (E/L), early-type spiral (ES), and late-type spiral galaxies (LS).

It was found that:

1) As was previously shown by other studies, the SN Ia stretch parameter is closely correlated with host morphology. The low stretch (fast decline) SNe usually exploded in E/L galaxies, i.e. in old stellar population environment (see Fig. 1).

2) No statistically significant trend for the colour parameter is found. 
3) There is a trend in the stretch nuisance parameter $\alpha$. Its value decreases from $E / L$ to LS galaxies.

4) In an old stellar population and with low dust environment, supernovae are intrinsically fainter after stretch and colour corrections. This conclusion contradicts the results obtained by [19] (see also [20] and references in it). However, our results are consistent within 1- $\sigma$ for all subsamples. This can be improved by adding SNLS JLA supernovae in the analysis.

5) The host galaxy morphology affects the residual dispersion in the Hubble diagram. It was noticed that SNe Ia in LS are more homogeneous with $R M S=0.115 \pm 0.010$.
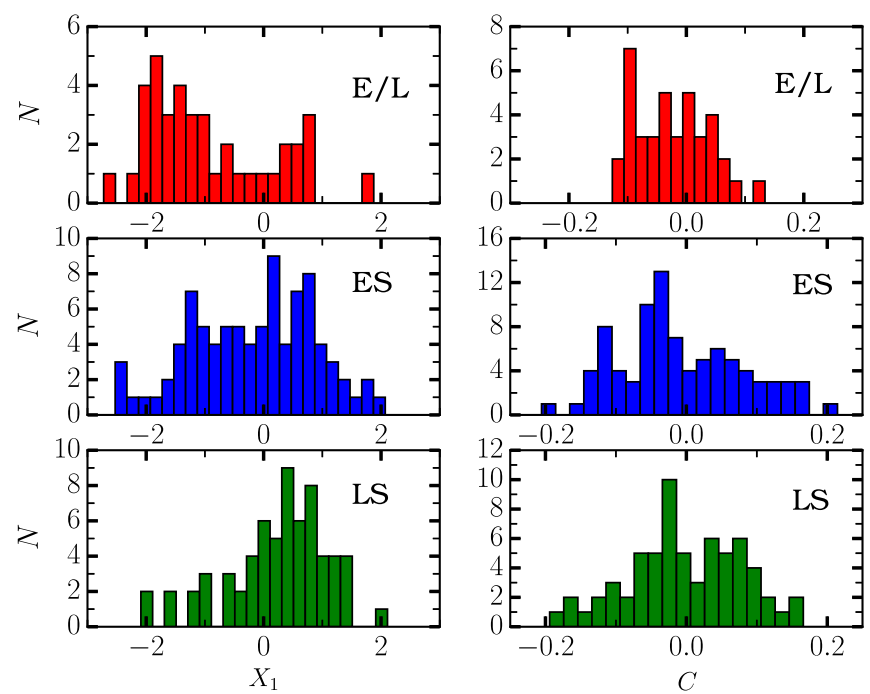

Figure 1: The distribution of the SALT2 parameters, stretch $X 1$ (left) and colour $C$ (right) for different host galaxy morphologies. The figure is taken from [1].

\section{Peculiar velocity correction}

To better estimate the cosmological redshift of the SNe Ia that were exploded in cluster of galaxies, the peculiar velocity correction can be introduced [2]. For a SN in a cluster it is possible to estimate $z_{c}$ more accurately using the host galaxy cluster redshift $\left(z^{c l}\right)$ instead of the host redshift $\left(z^{\text {host }}\right)$. The mean cluster redshift is not affected by virialization within a cluster. Of course clusters also have peculiar velocities which can sometimes manifest themselves as cluster merging, for example, the Bullet clusters [21]. However, clusters have much smaller peculiar velocities than the galaxies within them (i.e. $\sim 300 \mathrm{~km} \mathrm{~s}^{-1}$; [22, 23, 24]).

\subsection{Data}

As a parent sample 145 SNe Ia observed by the Nearby Supernova Factory (SNFACTORY), a project devoted to the study of SNe Ia in the nearby Hubble flow $(0.02<z<0.08$; [25]), were used. SNe Ia that appear to reside in known clusters of galaxies, were identified. Then, the impact of their peculiar velocities was estimated by replacing the host redshift by the cluster redshift. 
Using the SIMBAD database ([26]) we chose all the clusters projected within $\sim 2.5 \mathrm{Mpc}$ around the SNe Ia positions and with redshift differing from that of the supernova by less than 0.015. SN Ia host redshifts were used to initially determine the distance. We did not consider objects classified as groups of galaxies, although there is no strong boundary between these and clusters, since groups of galaxies are characterized by smaller mass and therefore smaller velocity dispersion $\sim 300 \mathrm{~km} \mathrm{~s}^{-1}$ (see fig. 5 in [27]). The uncertainty introduced by such velocity is properly accounted for using the conventional method of assigning a fixed uncertainty to all $\mathrm{SNe}$ Ia to account for peculiar velocities.

To perform the final matching between SN and host cluster, the redshift and the size of all host cluster candidates have to be known. First, we took all the galaxies attributed to each cluster in literature sources and added the SNFACTORY host galaxy if it was not among them. Then, these data were combined with the DR13 release database of SDSS [28, 29, 30, 31]. To characterize the cluster radius we used the virial radius $R_{200}$, corresponding to an average enclosed density equal to 200 times the critical density of the Universe at redshift z. To estimate the redshift of a cluster we applied the so-called bi-weight technique [32] on the remaining redshift distributions.

Finally, supernova is considered a cluster member if two conditions are satisfied:

- $r<R_{200}$, where $r$ is the projected distance between the $\mathrm{SN}$ and cluster center.

- $\left|z^{\text {host }}-z^{c l}\right|<3 \frac{\sigma_{V}}{c}$, where $\sigma_{V}$ is the velocity dispersion inside the cluster.

The SNe Ia that did not satisfy these criteria were removed from further consideration. For the remaining clusters we checked for the presence of X-ray emission(Fig. 3), a red sequence (Fig. 2) or the SZ effect.

The final list of SNFACTORY SNe Ia in confirmed clusters contains 11 objects. The resulting association of SNe Ia with host clusters is given in Table 1.

SNF20080731-000

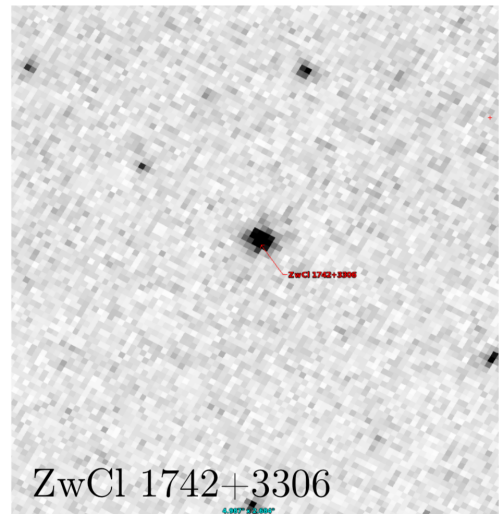

SN2007nq

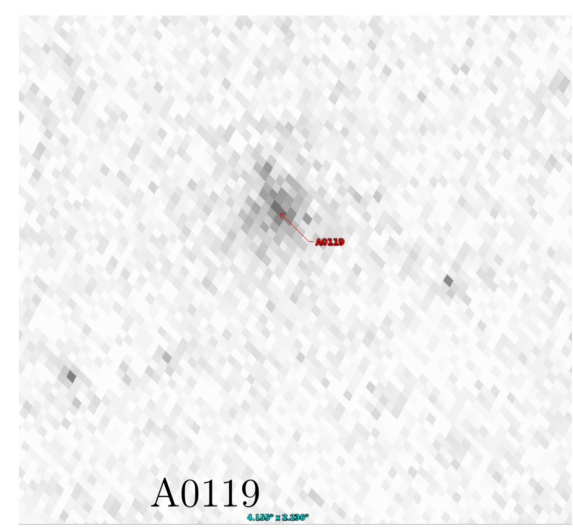

Figure 2: X-ray images for two galaxy clusters from our final sample of associations. We used the public ROSAT All Sky Survey images within the energy band 0.1-2.4 keV, to look for extended X-ray counterparts (http://www.xray.mpe.mpg.de/cgi-bin/rosat/rosat-survey). 

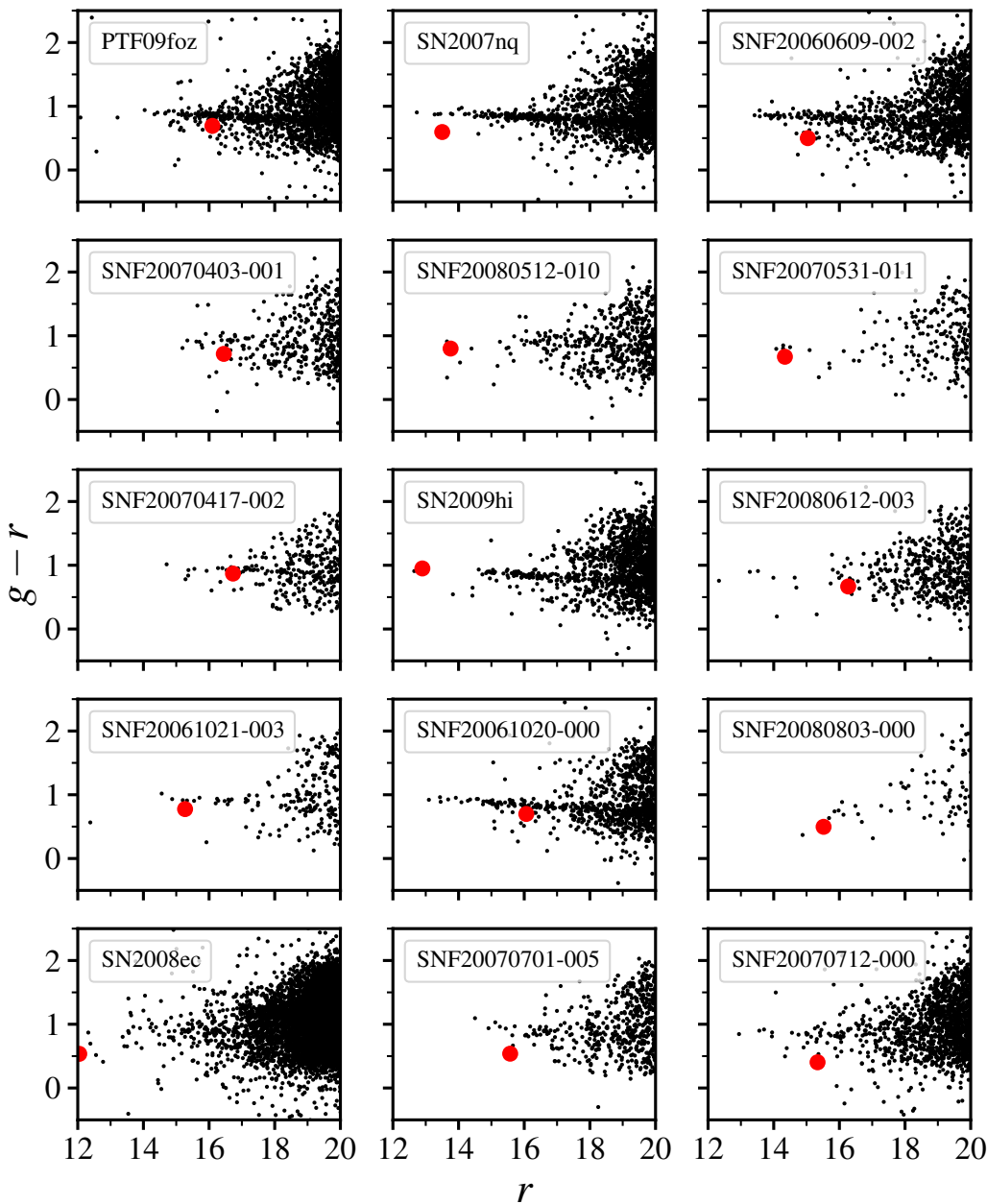

Figure 3: Color-magnitude diagram ( $g-r$ vs. $r$ ) plotted for the host clusters candidates for which SDSS galaxy redshifts and colors are available [28, 29, 30, 31]. Red points show the positions of supernova hosts, most of which are located near the red sequence.

\subsection{Hubble diagram}

Since we have a list of $11 \mathrm{SNe}$ Ia that belong to clusters, we can apply peculiar velocity correction and study how it affects the Hubble residuals. The following methodology is implemented.

The theoretical distance modulus is $\mu^{\text {th }}=5 \log _{10} d_{\mathrm{L}}-5$, where $d_{\mathrm{L}}$ is the true luminosity distance in parsecs:

$$
d_{\mathrm{L}}=\frac{c}{H_{0}}\left(1+z_{\mathrm{h}}\right) \int_{0}^{z_{c}} \frac{d z_{c}^{\prime}}{\sqrt{\Omega_{\Lambda}+\Omega_{m}\left(1+z_{c}^{\prime}\right)^{3}}}
$$

where $z_{\mathrm{h}}$ is the heliocentric redshift, which takes into account the fact that the observed flux is affected not only by the cosmological redshift but by the Doppler effect as well.

We assign the cosmological redshift $z_{c}$ to be: 


\begin{tabular}{llll}
\hline \hline SN Name & Host Cluster & MCXC Name & Cluster Coordinates \\
\hline SNF20051003-004/SN2005eu & RXJ0228.2+2811 & $\mathrm{J} 0228.1+2811$ & $022809.6+281140$ \\
SNF20060609-002 & A2151a & $\mathrm{J} 1604.5+1743$ & $160435.7+174328$ \\
SNF20061020-000 & A76 & $\mathrm{J} 0040.0+0649$ & $004000.5+064905$ \\
SNF20061111-002 & RXC J2306.8-1324 & $\mathrm{J} 2306.8-1324$ & $230651.7-132459$ \\
SNF20080612-003 & RXC J1615.5+1927 & $\mathrm{J} 1615.5+1927$ & $161534.7+192736$ \\
SNF20080623-001 & ZwCl8338 & $\mathrm{J} 1811.0+4954$ & $181100.1+495440$ \\
SNF20080731-000 & ZwCl 1742+3306 & $\mathrm{J} 1744.2+3259$ & $174415.0+325923$ \\
PTF09foz & A87/A85 & $\mathrm{J} 0041.8-0918$ & $004150.1-091807$ \\
SN2006X & Virgo & $\mathrm{J} 1230.7+1220$ & $123047.3+122013$ \\
SN2007nq & A119 & $\mathrm{J} 0056.3-0112$ & $005618.3-011300$ \\
SN2009hi & A2589 & $\mathrm{J} 2323.8+1648$ & $232353.5+164832$ \\
\hline
\end{tabular}

Table 1: The association of the SNFACTORY SNe Ia with host clusters [2].

$$
z_{c}=\left\{\begin{array}{l}
z_{c}^{c l} \quad \text { if inside a galaxy cluster } \\
z_{c}^{\text {host }} \text { otherwise }
\end{array}\right.
$$

The uncertainty on redshift measurement and peculiar velocity correction is assigned as:

$$
\sigma_{z}= \begin{cases}\frac{5 \sqrt{z_{\text {err }}^{c l}}}{z^{c l} \ln (10)} & \text { if inside a galaxy cluster, } \\ \frac{5 \sqrt{z_{\text {err }}^{\text {host }} 2+0.001^{2}}}{z^{\text {host }} \ln (10)} & \text { otherwise. }\end{cases}
$$

The 0.001 value corresponds to the $300 \mathrm{~km} \mathrm{~s}^{-1}$ that is added to the redshift error of SNe Ia outside the clusters in order to take into account the unknown galaxy peculiar velocities, as in a classical cosmological analysis. For cases where a SN Ia belongs to a galaxy cluster, we assume that the redshift error contains only the error due to the redshift measurement of a cluster.

In order to study the impact of peculiar velocity correction, we performed the Hubble diagram fit and computed the Hubble residuals for the SNe Ia in clusters before and after correction. We used the weighted root mean square (wRMS) as defined in [33] to measure the impact of this correction. We used the same intrinsic dispersion established during the fitting ( $\sigma_{\text {int }}=0.10 \mathrm{mag}$ ) to calculate all $w R M S$. SN2006X is not taken into account during the computation of the $w R M S$ due to the fact that it does not belong to the set of "normal" SNe Ia.

The dispersion of these $11 \mathrm{SNe}$ Ia around the Hubble diagram decreases significantly when the peculiar velocities of their hosts inside the clusters are taken into account ( $w R M S=0.130 \pm$ $0.038 \mathrm{mag}$ ). When using the redshift of the host instead of the redshift of the cluster, the dispersion of these $11 \mathrm{SNe}$ Ia is $w R M S=0.137 \pm 0.036 \mathrm{mag}$ (see Fig. 4). The significance of this improvement is $\sim 3.6 \sigma$.

Moreover, the properties of the galaxies inside the clusters could be different from those in the field. While in the field we observe all morphological types of the galaxies, in the central part of the clusters usually the elliptical galaxies are located. It means that SNe Ia could be physically 


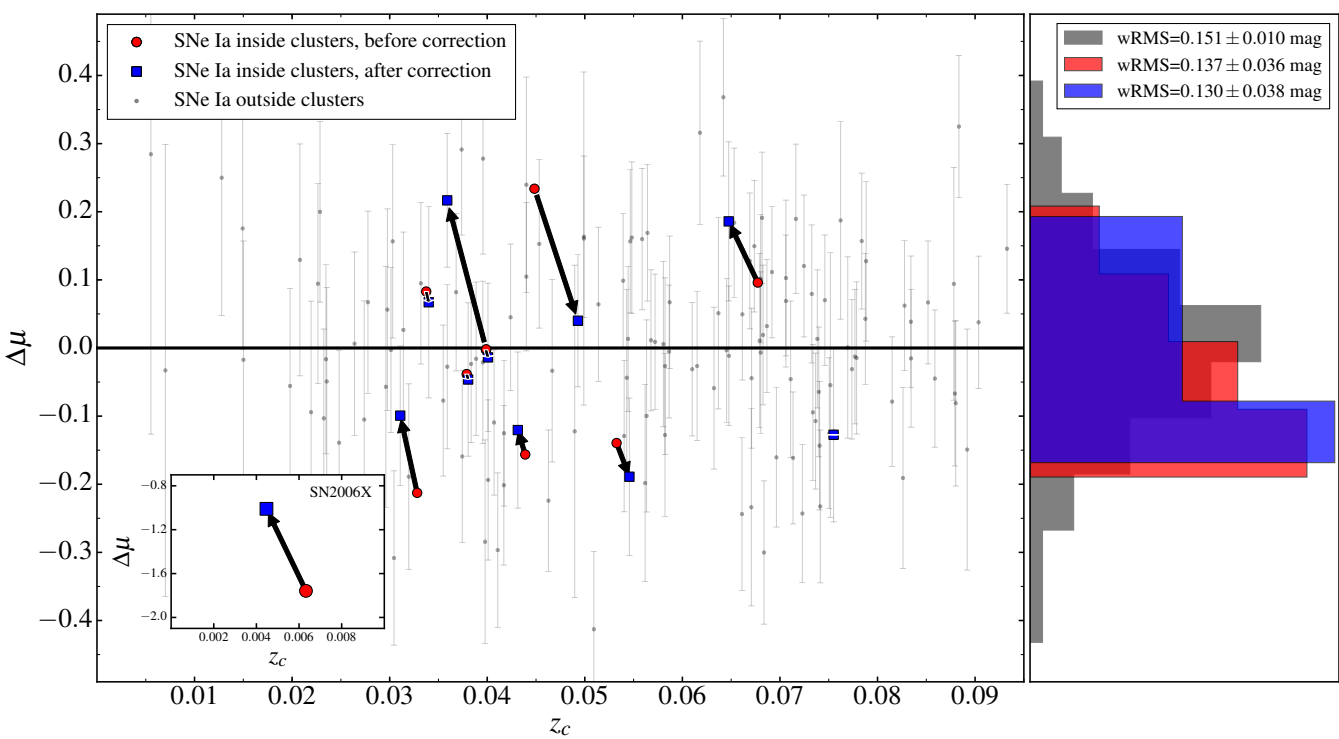

Figure 4: Hubble diagram residuals. For cluster members red circles (blue squares) and histograms correspond to residuals for SNe Ia in galaxy clusters before (after) correction for peculiar velocities of the hosts inside their clusters. The black histogram corresponds to all SNe Ia after correction. SN2006X is presented in the inset plot separately from the others due to its very large offset. The figure is taken from [2].

different inside and outside the clusters that can be crucial for future cosmological analysis where all supernovae are described by one standardization model. Indeed, the $w R M S$ we found for SNe Ia inside the clusters before correction, $0.137 \pm 0.036 \mathrm{mag}$, is also smaller than the $w R M S$ for the $\mathrm{SNe}$ Ia in the field $(w R M S=0.151 \pm 0.010 \mathrm{mag})$. However, our further analysis has shown that this difference is consistent with statistical fluctuations.

\section{Conclusions}

In this work we claim that a complex study of the impact of SN environment and peculiar velocities on the distance measurements and cosmology is needed.

Many studies shows that an environment plays an important role in the SN Ia standardization. The correlation between SN luminosity and host morphology was studied by [34, 1]; star formation rate [35, 36, 37, 38]; local environment [39, 40]; distance to galaxy center [41]; host stellar mass [35, 37]. However, all these studies were performed on nearby SN samples and have never been included in cosmological analyses. The first serious attempt to include the environmental factor in the Hubble diagram fitting was made in JLA analysis [14]. They introduced a simple correction for the host stellar mass as a step function in the standardization equation. Despite of such a rough procedure, it helped to decrease the spread on the Hubble diagram. However, the physical connection between environment and SN Ia luminosity is still unclear.

Another parameter that affects the Hubble diagram is unknown peculiar velocities. To better estimate the cosmological redshift of the SNe Ia that were exploded in cluster of galaxies, where the peculiar velocities of galaxies can be $>1000 \mathrm{~km} \mathrm{~s}^{-1}$, we introduced the peculiar velocity cor- 
rection and proposed to use the host galaxy cluster redshift instead of the host redshift. Using 145 SNe Ia from the Nearby Supernova Factory 11 candidates for membership in clusters were found. It was shown that for SNe Ia inside galaxy clusters the dispersion around the Hubble diagram when peculiar velocities are taken into account is smaller in comparison with a case without peculiar velocity correction, with a $w R M S=0.130 \pm 0.038$ mag instead of $w R M S=0.137 \pm 0.036$ mag [2]. However, this study was done with nearby sample of supernovae. The larger sample with higher redshifts is needed to measure the effect of peculiar velocity correction on redshift measurements with better accuracy and to estimate its possible impact on cosmology.

We are going to examine the peculiar velocity correction and "environmental" parameter using larger cosmological samples of SNe Ia (e.g., Pantheon sample [7]). Our final goal is to reduce as much as possible the residual dispersion on the Hubble diagram to improve the precision of cosmological parameters.

\section{Acknowledgments}

We thank the SNFACTORY collaboration for the supernovae dataset, their support and feedback. We also acknowledge the LSST group of Laboratoire de Physique de Clermont. Support in France was provided by CNRS/IN2P3. MVP acknowledges support from Russian Science Foundation grant 18-72-00159 for the selection of SNe exploded in galaxy clusters and study the impact of SN environment. PFL acknowledges support from the National Science Foundation grant PHY1404070.

\section{References}

[1] V. Henne, M. V. Pruzhinskaya, P. Rosnet, P.-F. Léget, E. E. O. Ishida, A. Ciulli et al., The influence of host galaxy morphology on the properties of Type Ia supernovae from the JLA compilation, New A $\mathbf{5 1}$ (Feb., 2017) 43-50, [1608.03674].

[2] P.-F. Léget, M. V. Pruzhinskaya, A. Ciulli, E. Gangler, G. Aldering, P. Antilogus et al., Correcting for peculiar velocities of Type Ia supernovae in clusters of galaxies, A\&A 615 (Aug., 2018) A162, [1804.03418].

[3] S. Perlmutter, G. Aldering, M. della Valle, S. Deustua, R. S. Ellis, S. Fabbro et al., Discovery of a supernova explosion at half the age of the universe, Nature 391 (Jan., 1998) 51, [astro-ph/9712212].

[4] S. Perlmutter, G. Aldering, G. Goldhaber, R. A. Knop, P. Nugent, P. G. Castro et al., Measurements of Omega and Lambda from 42 High-Redshift Supernovae, ApJ 517 (June, 1999) 565-586.

[5] A. G. Riess, A. V. Filippenko, P. Challis, A. Clocchiatti, A. Diercks, P. M. Garnavich et al., Observational Evidence from Supernovae for an Accelerating Universe and a Cosmological Constant, AJ 116 (Sept., 1998) 1009-1038.

[6] B. P. Schmidt, N. B. Suntzeff, M. M. Phillips, R. A. Schommer, A. Clocchiatti, R. P. Kirshner et al., The High-Z Supernova Search: Measuring Cosmic Deceleration and Global Curvature of the Universe Using Type IA Supernovae, ApJ 507 (Nov., 1998) 46-63, [astro-ph / 9805200 ].

[7] D. M. Scolnic, D. O. Jones, A. Rest, Y. C. Pan, R. Chornock, R. J. Foley et al., The Complete Light-curve Sample of Spectroscopically Confirmed SNe Ia from Pan-STARRSI and Cosmological Constraints from the Combined Pantheon Sample, ApJ 859 (June, 2018) 101, [1710.0 0845]. 
[8] B. W. Rust, Use of supernovae light curves for testing the expansion hypothesis and other cosmological relations, Ph.D. thesis, Oak Ridge National Lab., TN., 1974.

[9] I. P. Pskovskii, Light curves, color curves, and expansion velocity of type I supernovae as functions of the rate of brightness decline, Soviet Ast. 21 (Dec., 1977) 675-682.

[10] Y. P. Pskovskii, Photometric classification and basic parameters of type I supernovae, Soviet Ast. 28 (Dec., 1984) 658-664.

[11] M. Hamuy, M. M. Phillips, N. B. Suntzeff, R. A. Schommer, J. Maza and R. Aviles, The Hubble Diagram of the Calan/Tololo Type IA Supernovae and the Value of HO, AJ 112 (Dec., 1996) 2398, [astro-ph/9609062].

[12] R. Tripp, A two-parameter luminosity correction for Type IA supernovae, A\&A 331 (Mar., 1998) 815-820.

[13] N. Suzuki, D. Rubin, C. Lidman, G. Aldering, R. Amanullah, K. Barbary et al., The Hubble Space Telescope Cluster Supernova Survey. V. Improving the Dark-energy Constraints above $z>1$ and Building an Early-type-hosted Supernova Sample, ApJ 746 (Feb., 2012) 85, [1105. 3470 ].

[14] M. Betoule, R. Kessler, J. Guy, J. Mosher, D. Hardin, R. Biswas et al., Improved cosmological constraints from a joint analysis of the sdss-ii and snls supernova samples", A\&A 568 (2014) 32.

[15] J. Ruel, G. Bazin, M. Bayliss, M. Brodwin, R. J. Foley, B. Stalder et al., Optical Spectroscopy and Velocity Dispersions of Galaxy Clusters from the SPT-SZ Survey, ApJ 792 (Sept., 2014) 45, [1311.4953].

[16] J. Guy, P. Astier, S. Baumont, D. Hardin, R. Pain, N. Regnault et al., SALT2: using distant supernovae to improve the use of type Ia supernovae as distance indicators, A\&A 466 (Apr., 2007) 11-21.

[17] P.-F. Léget, Spectral modeling of type Ia supernovae, observed by the Nearby Supernova Factory, in order to improve extragalactic distance measurement, phd theses, Université Blaise Pascal, Sept., 2016.

[18] M. V. Pruzhinskaya, E. S. Gorbovskoy and V. M. Lipunov, ”Pure” supernovae and accelerated expansion of the Universe, Astronomy Letters 37 (Oct., 2011) 663-669, [1109.1159].

[19] M. Hicken, W. M. Wood-Vasey, S. Blondin, P. Challis, S. Jha, P. L. Kelly et al., Improved Dark Energy Constraints from 100 New CfA Supernova Type Ia Light Curves, ApJ 700 (Aug., 2009) 1097-1140, [0 901.4804$]$.

[20] M. Sullivan, A. Conley, D. A. Howell, J. D. Neill, P. Astier, C. Balland et al., The dependence of Type Ia Supernovae luminosities on their host galaxies, MNRAS 406 (Aug., 2010) 782-802, [1003.5119].

[21] D. Clowe, M. Bradač, A. H. Gonzalez, M. Markevitch, S. W. Randall, C. Jones et al., A Direct Empirical Proof of the Existence of Dark Matter, ApJL 648 (Sept., 2006) L109-L113, [astro-ph/0608407].

[22] N. A. Bahcall and S. P. Oh, The peculiar velocity function of galaxy clusters, The Astrophysical Journal Letters 462 (1996) L49.

[23] D. A. Dale, R. Giovanelli, M. P. Haynes, L. E. Campusano and E. Hardy, Seeking the local convergence depth. v. tully-fisher peculiar velocities for 52 abell clusters, The Astronomical Journal 118 (1999) 1489. 
[24] K. L. Masters, C. M. Springob, M. P. Haynes and R. Giovanelli, SFI++ I: A New I-Band Tully-Fisher Template, the Cluster Peculiar Velocity Dispersion, and $H_{0}, A p J 653$ (Dec., 2006) 861-880, [astro-ph/0609249].

[25] G. Aldering, G. Adam, P. Antilogus, P. Astier, R. Bacon, S. Bongard et al., Overview of the Nearby Supernova Factory, in SPIE Conference Series, vol. 4836, pp. 61-72, Dec., 2002.

[26] M. Wenger, F. Ochsenbein, D. Egret, P. Dubois, F. Bonnarel, S. Borde et al., The SIMBAD astronomical database. The CDS reference database for astronomical objects, A\&A, Supplement 143 (Apr., 2000) 9-22, [astro-ph/0002110].

[27] J. S. Mulchaey, X-ray Properties of Groups of Galaxies, Annual Review of Astron and Astrophys 38 (2000) 289-335, [astro-ph/0009379].

[28] D. J. Eisenstein, D. H. Weinberg, E. Agol, H. Aihara, C. Allende Prieto, S. F. Anderson et al., SDSS-III: Massive Spectroscopic Surveys of the Distant Universe, the Milky Way, and Extra-Solar Planetary Systems, AJ 142 (Sept., 2011) 72, [1101.1529].

[29] K. S. Dawson, D. J. Schlegel, C. P. Ahn, S. F. Anderson, É. Aubourg, S. Bailey et al., The Baryon Oscillation Spectroscopic Survey of SDSS-III, AJ 145 (Jan., 2013) 10, [1208 . 0 022].

[30] S. A. Smee, J. E. Gunn, A. Uomoto, N. Roe, D. Schlegel, C. M. Rockosi et al., The Multi-object, Fiber-fed Spectrographs for the Sloan Digital Sky Survey and the Baryon Oscillation Spectroscopic Survey, AJ 146 (Aug., 2013) 32, [1208.2233].

[31] SDSS Collaboration, F. D. Albareti, C. Allende Prieto, A. Almeida, F. Anders, S. Anderson et al., The Thirteenth Data Release of the Sloan Digital Sky Survey: First Spectroscopic Data from the SDSS-IV Survey MApping Nearby Galaxies at Apache Point Observatory, ArXiv e-prints (Aug., 2016), [1608.02013].

[32] T. C. Beers, K. Flynn and K. Gebhardt, Measures of location and scale for velocities in clusters of galaxies - A robust approach, AJ 100 (July, 1990) 32-46.

[33] S. Blondin, K. S. Mandel and R. P. Kirshner, Do spectra improve distance measurements of Type Ia supernovae?, A\&A $\mathbf{5 2 6}$ (Feb., 2011) A81.

[34] M. Sullivan, D. Le Borgne, C. J. Pritchet, A. Hodsman, J. D. Neill, D. A. Howell et al., Rates and Properties of Type Ia Supernovae as a Function of Mass and Star Formation in Their Host Galaxies, ApJ 648 (Sept., 2006) 868-883, [astro-ph/ 0605455 ].

[35] M. Sullivan, A. Conley, D. A. Howell, J. D. Neill, P. Astier, C. Balland et al., The dependence of type ia supernova luminosities on their host galaxies, 1003.5119.

[36] J. D. Neill, M. Sullivan, D. A. Howell, A. Conley, M. Seibert, D. C. Martin et al., The Local Hosts of Type Ia Supernovae, ApJ 707 (Dec., 2009) 1449-1465, [0911.0690].

[37] J. Johansson, D. Thomas, J. Pforr, C. Maraston, R. C. Nichol, M. Smith et al., SN Ia host galaxy properties from Sloan Digital Sky Survey-II spectroscopy, MNRAS 435 (Oct., 2013) 1680-1700, [1211.1386].

[38] H. Campbell, M. Fraser and G. Gilmore, How SN Ia host-galaxy properties affect cosmological parameters, MNRAS 457 (Apr., 2016) 3470-3491, [1602.02596].

[39] M. Rigault, Y. Copin, G. Aldering, P. Antilogus, C. Aragon, S. Bailey et al., Evidence of environmental dependencies of Type Ia supernovae from the Nearby Supernova Factory indicated by

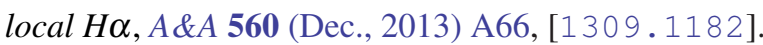


[40] M. Roman and E. Rauscher, Modeled Temperature-Dependent Clouds with Radiative Feedback in Hot Jupiter Atmospheres, ArXiv e-prints (July, 2018) , [1807. 08890].

[41] R. Hill, H. Shariff, R. Trotta, S. Ali-Khan, X. Jiao, Y. Liu et al., Projected distances to host galaxy reduce SNIa dispersion, ArXiv e-prints (Dec., 2016), [1612.04417]. 